\title{
Being Reactive: Assessing Affect in the COVID-19 Era
}

\author{
Matthew N. Goldenberg ${ }^{1}$ (D) - Jonathan S. Gerkin ${ }^{2} \cdot$ Kenan M. Penaskovic ${ }^{2}$
}

Received: 27 April 2020 / Accepted: 19 July 2020 / Published online: 5 August 2020

(C) Academic Psychiatry 2020

To the Editor:

The COVID-19 pandemic has dramatically impacted almost every sector of American society, including clinical psychiatry. Many psychiatrists have moved our practices to telehealth platforms, treating patients quarantined in their homes. Those of us still working on-site are often wearing masks, as are many of our patients. We have altered the structure and staffing of inpatient units, trying to promote physical distancing while still caring for the acutely ill. To limit exposure and conserve personal protective equipment, consultation-liaison psychiatrists are both "seeing" patients and offering recommendations by electronic device.

These changes have forced a reconsideration of a fundamental psychiatric skill-assessing affect. As we teach our students, affect is the observable expression of one's emotional state. As a core part of the mental status examination, we traditionally record a patient's affect: its quality, stability, range, intensity, and level of congruence with stated mood. This assessment incorporates a patient's facial expression, tone of voice, body language, content of speech, and interactive reciprocity. Our evaluation of affect is often subconscious and subtle, a skill we may largely take for granted. But we use this evaluation to inform our diagnosis, safety assessment, treatment recommendation, and monitoring of illness course.

How does one judge a patient's affect if her face is largely hidden by a mask? Her uncovered eyes may be helpful, but not fully. Not being able to see the lower part of a patients' face undoubtedly compromises our assessment. Is she smiling, sulking, smirking, or sneering? We use her volume and tone of voice as data, but those also may be affected by wearing the mask. Is she shouting because she is angry or just to ensure being heard? Is she notably quiet, responding to

Matthew N. Goldenberg

matthew.goldenberg@yale.edu

\section{Yale University School of Medicine, New Haven, CT, USA}

2 University of North Carolina School of Medicine, Chapel Hill, NC, USA internal stimuli, or just muffled by the cloth barrier? Moreover, to what extent is her affect influenced by the unfamiliarity of speaking with a masked psychiatrist?

Those of us now conducting patient interviews by phone (e.g., when a patient lacks access to a computer or smartphone and/or prefers phone contact) may encounter similar difficulties. Trust and empathy are potentially harder to establish with new patients. We find ourselves relying on non-visual clues, inferring affect primarily from tone and prosody of voice, perhaps from an expression or appreciation of humor. We may need to document differently: "Based on content-appropriate changes to rate/prosody/intonation of voice, the patient remains somewhat dysthymic yet reactive without significant lability, which is congruent with her mood."

Telepsychiatry visits that allow for both audio and visual contact, without masks, would seem to mitigate many of the above concerns. But telepsychiatric interviewing raises other considerations regarding assessment of affect. While some telehealth platforms may offer excellent audiovisual fidelity in general, there can at times be lags that affect the synchrony of voice and picture. Some patients, particularly those less familiar with the technology, may sport primarily concerned or inquisitive looks. Other patients seem to emote more via video than in person, possibly to ensure that their feelings are being appreciated. The affect of a person with paranoia may be influenced by suspicion about the technology itself, and he may be reluctant to engage in a telepsychiatric interview altogether.

As psychiatrists working in the era of COVID-19, it is our duty to help our current and future patients, our colleagues and our communities navigate through an increasingly uncertain world. To do so, we will need to be flexible, adapting not only to new ways of delivering care but also modifying how we conduct mental status examinations and interpret fundamental data such as affect.

Disclosures On behalf of all authors, the corresponding author states that there is no conflict of interest.

Publisher's Note Springer Nature remains neutral with regard to jurisdictional claims in published maps and institutional affiliations. 